\title{
Giant right pulmonary vein thrombus
}

\author{
Marco Rolando ${ }^{1}$, Giacomo Ingallina ${ }^{1}$, Mellone Renata ${ }^{1}$, Stefano Stella ${ }^{1}$, Francesco \\ Ancona $^{2}$, Cristina Capogrosso ${ }^{1}$, Francesco Melillo ${ }^{1}$, and Eustachio Agricola ${ }^{3}$ \\ ${ }^{1}$ San Raffaele Hospital \\ ${ }^{2}$ Ospedale San Raffaele \\ ${ }^{3}$ IRCCS S Raffaele
}

August 22, 2020

\begin{abstract}
Pulmonary Vein Thrombosis (PVT) is a rare and insidious condition with only few case series and case reports described in literature. PVT effects on pulmonary circulation is similar to mitral stenosis and its haemodynamic consequences depend on the number of veins involved, the obstruction severity and the pulmonary vessels compliance. Here we present a case in which a multimodality approach with Transesophageal Echocardiography (TEE) and Computed Tomography (CT) imaging helped to guide therapeutic decisions. Specifically, a post-processing reconstruction of TEE projection directly from CT imaging was performed.
\end{abstract}

\section{Giant right pulmonary vein thrombus}

Marco Rolando, $\mathrm{MD}^{1}$, Giacomo Ingallina, $\mathrm{MD}^{1}$, Renata Mellone, $\mathrm{MD}^{2}$, Stefano Stella, MD ${ }^{1}$, Francesco Ancona, $\mathrm{MD}^{1}$, Cristina Capogrosso, $\mathrm{MD}^{1}$, Francesco Melillo, $\mathrm{MD}^{1}$, Eustachio Agricola, $\mathrm{MD}^{1,3}$

${ }^{1}$ Unit of Cardiovascular Imaging, IRCCS San Raffaele Scientific Institute, Milan, Italy

${ }^{2}$ Department of Radiology and Experimental Imaging Center, IRCCS San Raffaele Scientific Institute, Milan, Italy

${ }^{3}$ Vita-Salute San Raffaele University, IRCCS San Raffaele Scientific Institute, Milan, Italy

\section{Running head: Giant pulmonary vein thrombus}

\section{Corresponding Author}

Dr. Giacomo Ingallina

Echocardiography Laboratory

San Raffaele Hospital, IRCCS

Via Olgettina 60, 20132 Milan, Italy

Tel. +3902 26437313 
Fax. +390226437358

e-mail: ingallina.giacomo@hsr.it

\section{Case report}

A 75-year-old female was referred to our hospital with a history of dedifferentiated acinic cell carcinoma of the left parotid gland treated with total parotidectomy and lymphadenectomy followed by radiation therapy.

For pulmonary metastases a chemotherapy with carboplatin and taxol was started. This treatment was suspended after three months for the occurrence of severe neutropenia and the identification of mild myelodysplastic syndrome. Computed tomography (CT) scan of the chest with iodinated contrast was performed for staging and a partial filling defect of the right superior pulmonary vein (RSPV) related to a large thrombotic formation $(50 \times 20 \mathrm{~mm})$ protruding in left atrium (LA) and connected with pathological tissue of the pulmonary hilum (Figure 1) was found. She then started an anticoagulant therapy with Edoxaban 60 qd.

Four months later she started suffering from headache, diplopia and bilateral scotoma. During the evaluation in our emergency department, she was on sinus rhythm and the echocardiography showed heart chambers with normal function and dimensions without significant valvopathies. Her blood tests were within normal limits, except for a slight elevation of serum creatinine $(1.15 \mathrm{mg} / \mathrm{dl}$, estimated GFR $46 \mathrm{ml} / \mathrm{min})$. Plasma Edoxaban dosage $(188 \mathrm{ng} / \mathrm{ml}$ (RV $19.0-62.0 \mathrm{ng} / \mathrm{ml})$ confirmed the therapeutic range of the drug. Brain CT and MRI scan indicated multiple cerebral ischemic lesions (Figure I in Data Supplement).

In order to reassess the Pulmonary Vein Thrombus (PVT) after the anticoagulant therapy Transesophageal Echocardiography (TEE) was performed. The TEE confirmed a large RSPV thrombus protruding in LA as an oscillating shaggy hypoechoic mass of 50 x $12 \mathrm{~mm}$ (Figure 2a and Figure II in Data Supplement).

The Three-dimensional echocardiography clearly showed the thrombus origin from RSPV, its relationship with the other structures of LA and its multi-lobular aspect with "papillary-like" shape (Figure 3,Figure III and Video I-III in Data supplement). These characteristics proved the high embolic potential of the thrombus.

A direct comparison of the thrombus between the TEE and the CT scan performed four months earlier was performed thought reconstruction of the transesophageal projection from the CT dataset (Figure $2 \mathrm{~b}, 2 \mathrm{c}$ ).

This comparison demonstrated that the thrombus was not reduced after four months of anticoagulant therapy.

Thrombus removal with surgical intervention was excluded due to the patient's overall conditions. The anticoagulation therapy was switched from Edoxaban $60 \mathrm{mg}$ qd to Dabigatran $150 \mathrm{mg}$ bid, which demonstrated the greatest absolute reduction in risk of stroke compared to warfarin in atrial fibrillation. After two weeks of hospitalization, the patient was discharged with complete neurological recovery.

\section{Discussion}

Pulmonary vein thrombosis (PVT) is a rare and insidious condition in which most patients are usually asymptomatic or with non-specific symptoms [1-4]. Its real incidence is unknown, as literature includes only case report and case series.

The most frequent risk factors are primary and metastatic lung carcinoma [5-7], major lung surgery and chest trauma [1]. PVT was also described in association with atrial fibrillation $[2,6]$, polycitemia vera, treatment 
of asthma with omalizumab, autonomic nervous system dysfunction and various kind of neoplasia $[1,3,4]$. Sporadic cases were described as idiopathic [1].

The effect of a PVT on pulmonary circulation could be similar to that caused by a mitral stenosis, but it depends on the number of veins involved, the obstruction severity and the pulmonary vessels compliance.

The clinical presentation of PVT is a triad of cough, dyspnea and hemoptysis [1]. When the disease involves more than one pulmonary vein patients arrive at medical attention for pulmonary edema [1] and if only one side is affected a unilateral pulmonary edema can be observed.

To the best of our knowledge, this is the first case in which a PVT evolution was investigated using comprehensive TEE associated with CT scan and multimodality post-processing tools.

The PVT was identified as an incidental finding in a staging CT scan of the chest and after four months she had a stroke despite anticoagulant therapy.

After multidisciplinary consultation it was decided to re-evaluate the patient with TEE to better characterize the suspected thrombus after four months with anticoagulant therapy $[3,7]$.

The multimodality imaging approach is helpful to evaluate intracardiac masses. In particular, the CT scan with the ability to image in any plane allows full visualization of the heart and mediastinum that is crucial to identify origin, extension, relationship of the masses with nearby structures $[6,8]$.

The Real-time three-dimensional Echocardiographic assessment is useful to analyze the consistency, mobility and hemodynamic effects of masses [3,7].

Furthermore, the possibility of reconstructing TEE projection directly from CT imaging allows a direct comparison between the two techniques.

In this case this reconstruction enabled the comparison between the TEE images and the previous CT scan confirming that the thrombus was almost unchanged. This finding showed that anticoagulant therapy was ineffective and the stroke had cardioembolic origin.

\section{References}

1. Chaaya G, Vishnubhotla P. Pulmonary vein thrombosis: a recent systematic review. Cureus 2017; 9:e993-8.

2. Malm B, Hull S, Jadbabaie F: Left upper pulmonary vein thrombus in a patient with atrial fibrillation and prior lobectomy. Am J Med 2014; 127:7-8.

3. Kinsella JA, MacCarthy AJ, Kiernan TJ, Moore DP, McDermott RS, McCabe DJH. Transesophageal Echocardiographically-Confirmed Pulmonary Vein Thrombosis in Association with Posterior Circulation Infarction. Case Rep Neurol 2010, 2(1): 24-31.

4. Paul D. Stein, James E. Denier, Lawrence R. Goodman, Fadi Matta, Mary J. Hughes. Pulmonary vein thrombosis in patients with medical risk factors. Radiology Case Reports 2018; 1170-1173.

5. Chan V, Neumann D. Small cell lung carcinoma invading the pulmonary vein and left atrium as imaged by PET/CT. Eur J Nucl Med Mol Imaging. 2005; 32(12): 1493.

6. Porres DV, Morenza OP, Pallisa E, et al. Learning from the pulmonary veins. Radiographics, 2013; 33: $999-1022 \mathrm{~A}$

7. Kim NH, Roldan CA, Shively BK. Pulmonary vein thrombosis. Chest, 1993; 104(2): 624-6.

8. Takeuchi H. Diagnosis of a left lower pulmonary vein thrombus by 64-MDCT. BMJ Case Rep, 2013.

\section{Figure captions}

Figure 1. Chest CT scan axial slices demonstrating a perfusion defect (yellow arrows) in RSPV compatible with a thrombus protruding in left atrium. 
Figure 2. Transesophageal echocardiography and fusion imaging reconstruction. A) Biplane midesophageal view: right pulmonary vein view (left panel) and derived orthogonal view (right panel) showing a large mass originating from the RSPV. B) Reconstruction of virtual transesophageal views from CT dataset. These images were obtained by tracing the esophagus (dotted green line) in the CT thorax scan dataset. Left and right panel reproduce the corresponding echocardiographic image above allowing to identify the hyperdense formation originating from the RSPV (yellow arrows) and protruding into the LA. C) Volume rendering and the angiographic fusion imaging reconstruction of the virtual transesophageal study.

Figure 3. Three-dimensional echocardiographic reconstruction of LA displayed from "en-face" perspective showing the origin of the thrombotic formation from RSPV close to interatrial septum.

Supplemental Material Figure I: Multiple ischemic cerebral lesions (yellow arrows) at brain CT and MRI scans.

Supplemental Material Figure II: Transesophageal echocardiographic medioesophageal RSPV modified view (left panel) and the same acquisition (right panel) with real-time orthogonal plane.

Supplemental Material Figure III: Transesophageal three-dimensional reconstruction of LA from an "en-face" view (right panel) and the same image obtained with volume rendering reconstruction from the CT scan acquisition (left panel) with the thrombus (yellow arrows).

Video I: Transesophageal echocardiographic medioesophageal RSPV modified view with real-time orthogonal plane.

Video II: Transesophageal three-dimensional reconstruction focus on the thrombus.

Video III: Transesophageal three-dimensional reconstruction with thrombus from an "en-face" view. 


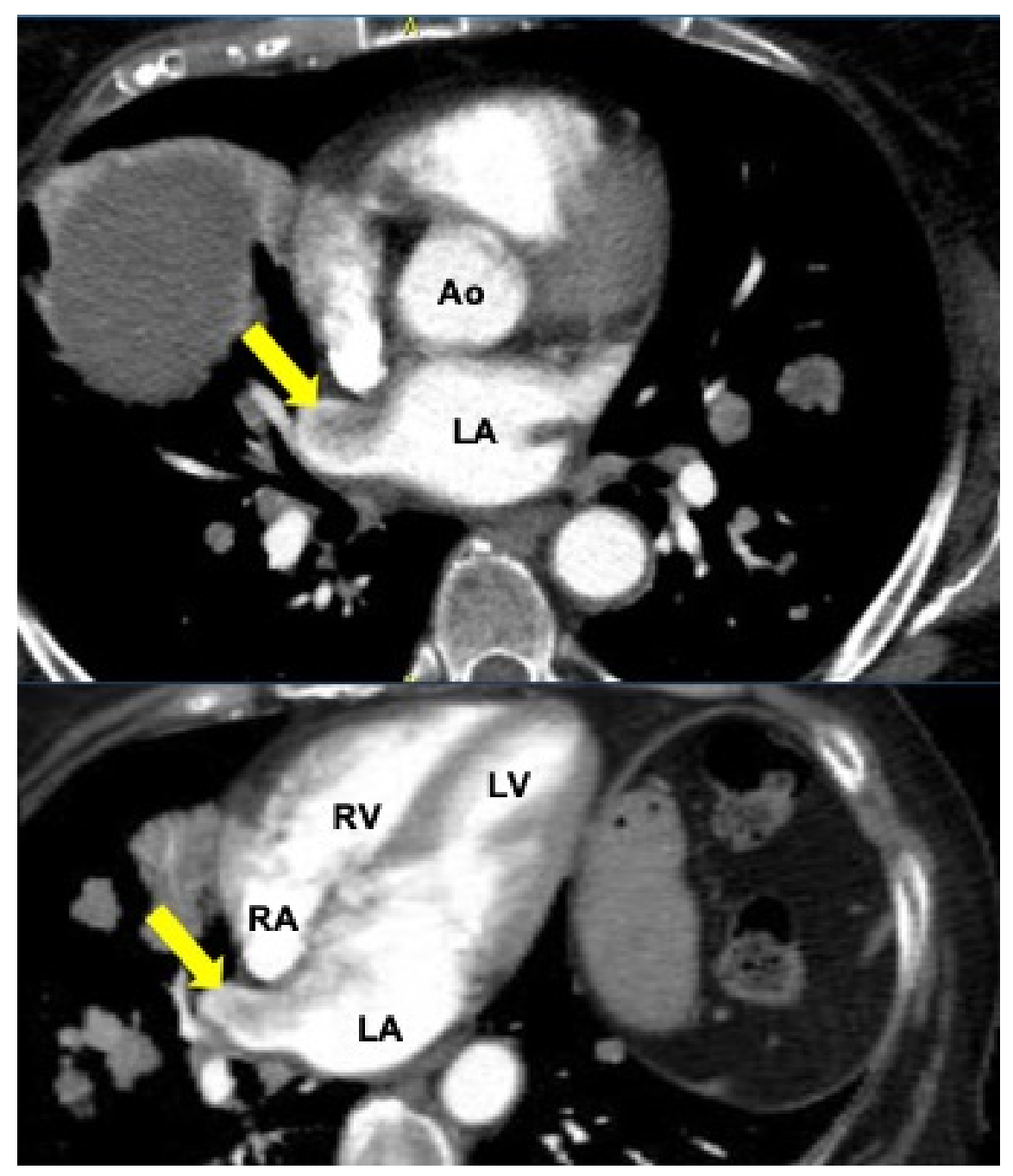




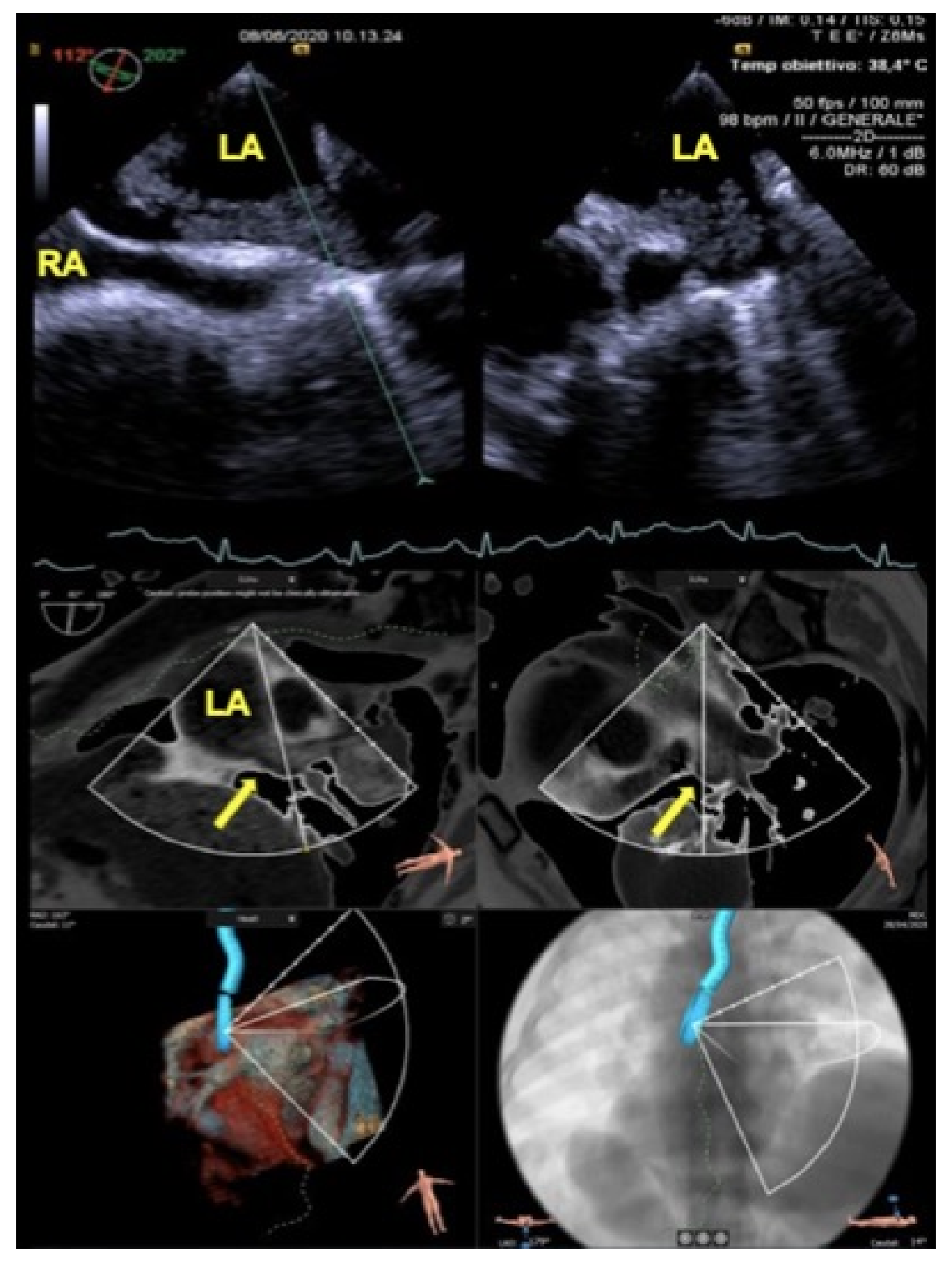




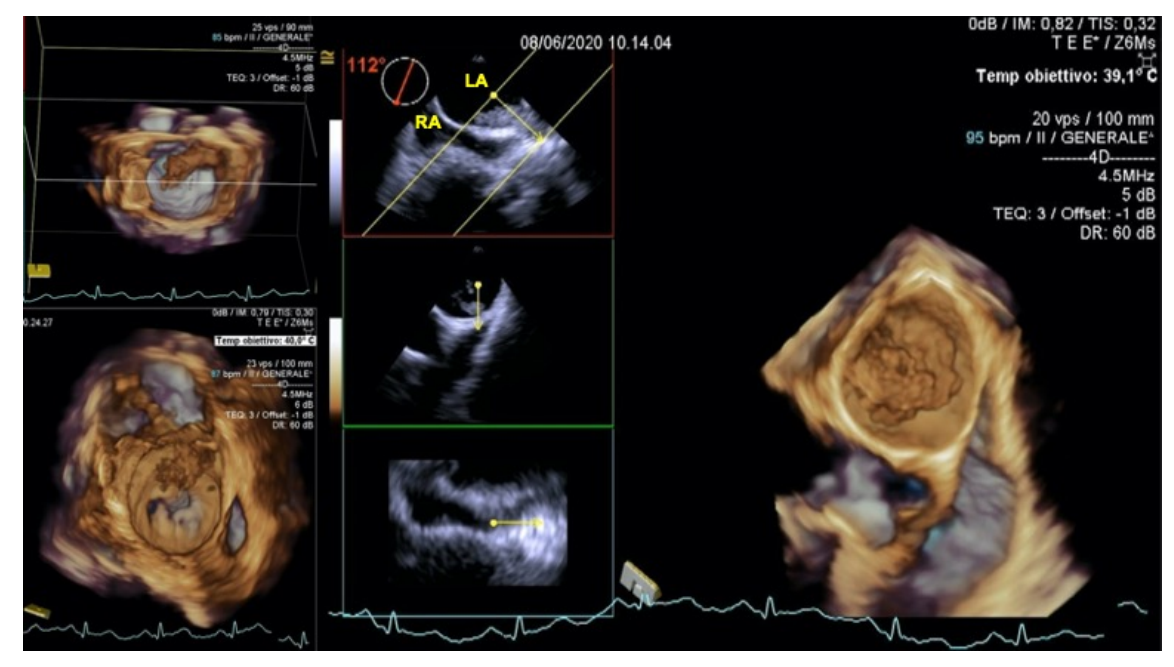

